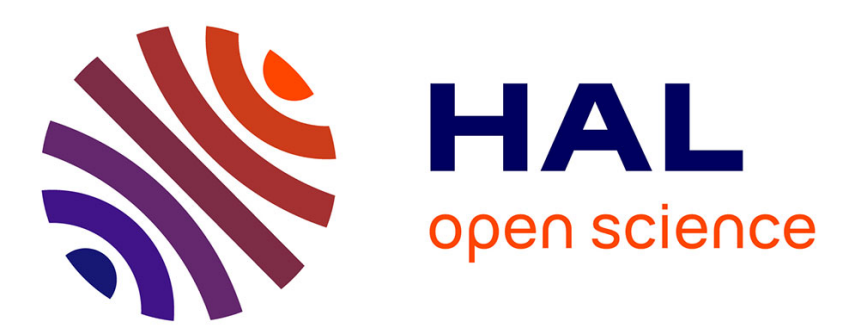

\title{
Power Loss in Reverberation Chambers by Antennas and Receivers
}

\author{
Andrea Cozza
}

\section{To cite this version:}

Andrea Cozza. Power Loss in Reverberation Chambers by Antennas and Receivers. IEEE Transactions on Electromagnetic Compatibility, 2017, 60 (6), pp.2041 - 2044. 10.1109/TEMC.2017.2777405 . hal01630674

\section{HAL Id: hal-01630674 \\ https://hal-centralesupelec.archives-ouvertes.fr/hal-01630674}

Submitted on 7 Nov 2017

HAL is a multi-disciplinary open access archive for the deposit and dissemination of scientific research documents, whether they are published or not. The documents may come from teaching and research institutions in France or abroad, or from public or private research centers.
L'archive ouverte pluridisciplinaire HAL, est destinée au dépôt et à la diffusion de documents scientifiques de niveau recherche, publiés ou non, émanant des établissements d'enseignement et de recherche français ou étrangers, des laboratoires publics ou privés. 


\title{
Power Loss in Reverberation Chambers by Antennas and Receivers
}

\author{
Andrea Cozza, Senior Member, IEEE
}

\begin{abstract}
Antennas, and more generally any equipment under test (EUT) capable of coupling to impinging electromagnetic waves, are well-known sources of power loss in reverberation chambers (RCs). As such, it is fundamental to model their contribution to an RC quality factor. The standard model available in the literature is based on power dissipated in loads connected to an antenna. This paper argues about the inaccuracy of this description, where internal dissipation within an antenna (or EUT), associated to low radiation efficiencies, is neglected. An alternative model is validated by showing its selfconsistency in predicting change in loading effects as opposed to the standard model. It is expected to have practical importance for tests performed in the low-frequency range of RCs, where antenna-related dissipation is the dominant loss mechanism, and in particular for EUTs which can be expected to display poor radiation efficiencies.
\end{abstract}

Index Terms-Reverberation chamber, quality factor, antenna coupling, power dissipation, radiation efficiency.

\section{INTRODUCTION}

Coupling to reverberation chambers (RCs) necessarily passes through antennas, acting as gateways for microwave power, especially so for injecting energy into RCs that will build up into high-intensity fields. Their typically reciprocal nature means that they also receive power at the same time, with the same effectiveness as they radiate it into RCs, causing power leakage which is typically dissipated into external loads. But antennas do not simply behave as gateways: they may introduce their own power dissipation mechanism, measured by their radiation efficiency $\eta_{a}$. These observations also apply to any receiver or EUT [1].

Since they contribute to the overall quality factor of an $\mathrm{RC}$, it is fundamental to know how to model their effect. The standard model available for predicting antenna-related losses was derived in [1]. Its main drawback is that it regards as lost only the power dissipated onto antenna loads, a description here argued to hold only for lossless antennas. Antenna mismatch and radiation efficiency do appear in the standard model, but they only modulate the power impinging on the load, neglecting the fact that radiation efficiency is a measure of internal power dissipation. Furthermore, reflection from mismatched loads inevitably leads to a second lossy interaction with the antenna.

This paper presents an improved model for antenna-related losses, taking into account these observations. The accuracy of the proposed model is demonstrated by its self-consistency in predicting change in power loss when different loads are connected to antennas. The two models disagree for weak

A. Cozza is with PIEM, GeePs (UMR 8507), 11 rue Joliot-Curie, 91192 Gif-sur-Yvette, France. Contact e-mail: andrea.cozza@ieee.org. radiation efficiencies, with the proposed model predicting greater loading effects. This latter case can be expected for imperfectly shielded EUTs, which are not designed to act as efficient antennas. Applications also involve predicting RC loading in their lower frequency range, where cavity-related losses are no longer the dominant loss mechanism.

\section{POWER TRANSMisSiOn THROUGH AN RC}

The average-power transmission $\sigma^{2}(\nu)$ of an RC measures the average power $\left\langle P_{o}(\nu)\right\rangle$ at the output port of a receiving antenna, as a function of frequency $\nu$, when an available power $P_{i}(\nu)$ is applied to the input port of a transmitting antenna, assumed to be identical to the receiving one, with no loss of generality. Brackets stand for the ensemble average.

The above definition corresponds to taking the variance of the $S_{21}(\nu)$ scattering parameter as measured, e.g., by a vector network analyzer (VNA), hence

$$
\sigma^{2}(\nu)=\frac{\left\langle P_{o}(\nu)\right\rangle}{P_{i}(\nu)}=\left\langle\left|S_{21}(\nu)\right|^{2}\right\rangle,
$$

having assumed diffusive conditions, hence $\left|\left\langle S_{21}(\nu)\right\rangle\right|^{2} \ll$ $\left\langle\left|S_{21}(\nu)\right|^{2}\right\rangle$.

The actual power injected into the RC is not $P_{i}(\nu)$, but rather $P_{t}(\nu)=P_{i}(\nu) \eta_{a}(\nu)\left(1-\left|\Gamma_{L}(\nu)\right|^{2}\right)$, with $\eta_{a}(\nu)$ the radiation efficiency of the antennas and $\Gamma_{L}(\nu)$ the impedance mismatch between the antennas impedances and their loads. Similarly, the output antenna receives on average a power $\left\langle P_{o}(\nu)\right\rangle=\left\langle P_{r}(\nu)\right\rangle \eta_{a}(\nu)\left(1-\left|\Gamma_{L}(\nu)\right|^{2}\right)$, with $\left\langle P_{r}(\nu)\right\rangle$ the average power available to the receiving antenna.

As shown in [1], $\sigma^{2}(\nu)$ can be expressed as a function of the quality factor $Q(\nu)$,

$$
\sigma^{2}(\nu)=\frac{Q(\nu)}{Q_{a}^{o}(\nu)} \eta_{a}^{2}\left(1-\left|\Gamma_{a}(\nu)\right|^{2}\right)^{2},
$$

where

$$
Q_{a}^{o}(\nu)=16 \pi^{2} V_{\lambda}
$$

accounts for power leaked by a perfectly matched and lossless antenna, expressed as a quality factor [1]; $V_{\lambda}=V / \lambda^{3}$ is the electric volume of the RC, with $\lambda$ the wavelength. Eq. (2) assumes that $\Gamma_{L}(\nu) \equiv \Gamma_{a}(\nu)$, i.e., the free-space reflection coefficient of the antennas, since the two RC-coupling antennas are usually connected to matched measuring devices, such as a VNA.

Antenna losses also appear in $Q(\nu)$ as [1]

$$
Q^{-1}(\nu)=M Q_{a}^{-1}(\nu)+Q_{c}^{-1}(\nu),
$$

where $Q_{c}(\nu)$ is the cavity-related quality factor, better known as intrinsic quality factor, while $Q_{a}(\nu)$ accounts for antennarelated losses. $M$ is the equivalent number of antennas, which 


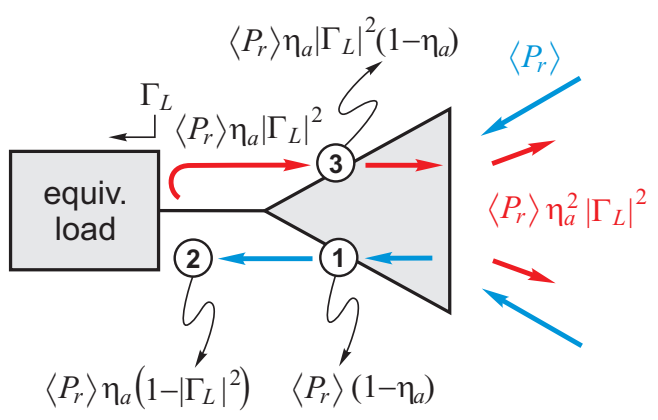

Fig. 1: Contributions to power dissipated by a loading antenna, with events related to received power in blue, and those leading to power radiated back to the $\mathrm{RC}$ in red. Three dissipation events marked from 1 to 3 are detailed in the text.

needs to be increased by one due to coherent backscattering for the input antenna, leading to a twofold increase in the power leaking through it [2], [3]. Hence, the case of two coupling antennas requires $M=3$.

The relation between $Q_{a}(\nu)$ and $Q_{a}^{o}(\nu)$ was also derived in [1], following the same reasoning used in expressing the injected power $P_{t}(\nu)$ as a function of the input power $P_{i}(\nu)$, thus

$$
Q_{a}(\nu)=\frac{Q_{a}^{o}(\nu)}{\eta_{a}(\nu)\left(1-\left|\Gamma_{L}(\nu)\right|^{2}\right)},
$$

by considering the antennas only leaking power outside the $\mathrm{RC}$; a generic $\Gamma_{L}(\nu)$ is now considered. Thus, a weakly efficient antenna is regarded as ineffective at leaking, thus justifying a higher $Q_{a}(\nu)$ that would make it weight less in (4).

\section{Power Dissipated By AN ANTENnA/EUT}

The standard definition of $Q_{a}(\nu)$, as reported in (5) is here shown to be inaccurate, since it neglects two fundamental steps in the description of antenna-related power loss. Taking the example of the receiving antenna in the previous section, $\left\langle P_{o}(\nu)\right\rangle$ only represents the fraction of available received power $\left\langle P_{r}(\nu)\right\rangle$ dissipated into the antenna load. Even though radiation efficiency and impedance mismatch are taken into account, they are only considered from the point of view of the antenna load. In fact, for $\eta_{a}(\nu)<1,\left\langle P_{r}(\nu)\right\rangle\left(1-\eta_{a}(\nu)\right)$ represents the fraction of available received power dissipated internally by the antenna, due to ohmic losses. This source of lost power is currently not acknowledged in (5), being distinct from the power dissipated into the antenna load.

For an antenna in receiving mode, the average available power $\left\langle P_{r}(\nu)\right\rangle$ undergoes a sequence of dissipative events illustrated in Fig. 1, marked as $1 \rightarrow 3$. Power is dissipated internally in 1 , already described, as $\left\langle P_{r}(\nu)\right\rangle\left(1-\eta_{a}(\nu)\right)$, leaving $\left\langle P_{r}(\nu)\right\rangle \eta_{a}(\nu)$ to impinge on the antenna load, of which only $\left\langle P_{r}(\nu)\right\rangle \eta_{a}\left(1-\left|\Gamma_{L}(\nu)\right|^{2}\right)$ is dissipated into the load, marked as 2 , the contribution acknowledged in (5). But for a general reflection coefficient $\Gamma_{L}(\nu)$, part of this impinging power wave will be subsequently reflected back and be available for the antenna now in transmission mode. This amounts to $\left\langle P_{r}(\nu)\right\rangle \eta_{a}(\nu)\left|\Gamma_{L}(\nu)\right|^{2}$, and will therefore be submitted again to internal dissipation within the antenna, with a third contribution to dissipation equal to $\left\langle P_{r}(\nu)\right\rangle \eta_{a}(\nu)\left(1-\eta_{a}(\nu)\right)\left|\Gamma_{L}(\nu)\right|^{2}$, while $\left\langle P_{r}(\nu)\right\rangle \eta_{a}^{2}(\nu)\left|\Gamma_{L}(\nu)\right|^{2}$ will be effectively radiated back into the RC.

In the end, the total dissipation as due to the sum of the three contributions described insofar corresponds to $\left\langle P_{r}(\nu)\right\rangle(1-$ $\left.\eta_{a}^{2}(\nu)\left|\Gamma_{L}(\nu)\right|^{2}\right)$, i.e., the portion of available received power not radiated back. As a result, (5) should be updated to

$$
Q_{a}(\nu)=\frac{Q_{a}^{o}(\nu)}{1-\eta_{a}^{2}(\nu)\left|\Gamma_{L}(\nu)\right|^{2}} .
$$

Since (6) was derived by including two additional contributions to dissipated power, (5) is necessarily a higher bound to (6). In other words, the standard model underestimates the contribution of antennas to dissipation phenomena in an RC. An example should help in understanding this situation. Consider a poorly efficient antenna: the available received power would be mostly dissipated within the antenna during two interactions, first in receiving mode and then when transmitting the power reflected by the load. This scenario is of practical importance, since $\eta_{a}(\nu) \simeq 1$ only for devices designed to radiate efficiently, i.e., antennas. But, as rightly pointed out in [1], models such as (5) and, as a consequence (6), also hold for any EUT capable of coupling to electromagnetic radiation. In this case, though, an EUT is usually not design to radiate, resulting in a likely low radiation resistance that may lead to a low radiation efficiency. The extreme case of $\eta_{a}(\nu)=0$ corresponds to complete dissipation in (6), while in (5) it is rather predicted to be vanishing.

The proposed model also displays an interesting symmetry between the role of $\eta_{a}(\nu)$ and of $\left|\Gamma_{L}(\nu)\right|$, as opposed to (5). An intuitive understanding of the reason for this symmetry can be gained by considering the case of an antenna with its output port connected to an attenuator terminated by a load with a mismatch $\Gamma_{L}(\nu)$. If $A(\nu)$ is the power attenuation, this setup can be substituted by two alternative equivalent circuits. In the first case, the antenna sees a load that now has an equivalent power reflection coefficient $\left|\Gamma_{L}(\nu)\right|^{2} A^{2}(\nu)$. Alternatively, the attenuator can be paired with the antenna, resulting in an apparent radiation efficiency $\eta_{a}(\nu) A(\nu)$, while maintaining the load reflection coefficient $\Gamma_{L}(\nu)$. The two equivalents must yield the same results dissipation-wise, with the same $Q_{a}(\nu)$. Eq. (6) complies with this physical requirement, thanks to the symmetry of its denominator. As a result, the case of a perfectly matched antenna or of a low radiation efficiency have the same effect, resulting into a strong dissipation and thus $Q_{a}(\nu) \simeq Q_{a}^{o}(\nu)$.

\section{VALIDATION PROCEDURE}

The validity of (6) cannot be tested directly, since $Q_{a}(\nu)$ cannot be estimated from experimental data without knowledge of the radiation efficiency of an antenna, while any estimate of $Q(\nu)$ assumes a negligible impact of antennas to an RC loading [4], [5].

One way of solving this conundrum is to compare predictions issued from the two models. Suppose $N$ further antennas were added to an RC, identical to the two used for measuring power transmission. Their power dissipation 
would be manifest as the average-power transmission through the RC would change, depending on the loads applied to the antennas port. An accurate model of antenna dissipation must then provide consistent predictions of their dissipation, independently of their loads. The accuracy of (5) and (6) requires that $\eta_{a}(\nu)$ estimated from power transmission through an RC be independent from the loads connected to the $N$ loading antennas.

Concretely, this amounts to take two sets of measurements of $\sigma^{2}(\nu)$, as defined in (1): first with no loading antenna $\left(\sigma_{o}^{2}(\nu)\right)$, and compare the results with the case when $N$ antennas were introduced into the RC $\left(\sigma_{N}^{2}(\nu)\right)$. From (2), $\sigma_{N}^{2}(\nu)$ is linked to the RC quality factor $Q_{N}(\nu)$, having updated (4) with the contributions from the $N$ loading antennas,

$$
Q_{N}^{-1}(\nu)=M Q_{a}^{-1}(\nu)+Q_{c}^{-1}(\nu)+N Q_{L}^{-1}(\nu),
$$

with $Q_{L}(\nu)$ the quality-factor contribution of the $N$ loading antennas closed on loads with reflection coefficient $\Gamma_{L}(\nu)$, whereas $Q_{a}(\nu)$ assumes $50 \Omega$ loads connected to the RCcoupling antennas. Then

$$
N Q_{L}^{-1}(\nu)=Q_{N}^{-1}(\nu)-Q_{o}^{-1}(\nu) .
$$

Recalling (2) and (6), it is possible to recast (8) as

$$
N\left(1-\eta_{a}^{2}(\nu)\left|\Gamma_{L}(\nu)\right|^{2}\right)=\eta_{a}^{2}(\nu)\left(1-\left|\Gamma_{a}(\nu)\right|^{2}\right)^{2} \Delta(\nu),
$$

where $\Delta(\nu)=1 / \sigma_{N}^{2}(\nu)-1 / \sigma_{o}^{2}(\nu)$ measures additional loss from the $N$ loading antennas.

Solving for $\eta_{a}(\nu)$, yields

$$
\eta_{a}^{2}(\nu) / N=\left[\left(1-\left|\Gamma_{a}(\nu)\right|^{2}\right)^{2} \Delta(\nu)+N\left|\Gamma_{L}(\nu)\right|^{2}\right]^{-1} .
$$

Similarly, the standard model (5) founds

$$
\eta_{a}^{2}(\nu) / N=\frac{1-\left|\Gamma_{L}(\nu)\right|^{2}}{\left(1-\left|\Gamma_{a}(\nu)\right|^{2}\right)^{2} \Delta(\nu)} .
$$

\section{EXPERIMENTAL RESULTS}

Models (10) and (11) bind the variation in power transmission $\Delta(\nu)$, due to antenna loading, with their termination impedance, through the fact that antenna radiation efficiency must be invariant to loading conditions. Therefore it makes for a good test of self-consistency, by comparing results obtained with multiple terminations $\Gamma_{L}(\nu)$.

Tests were carried out in an RC in CentraleSupelec, of dimensions $3.06 \times 1.84 \times 2.45 \mathrm{~m}^{3}$, equipped with a mechanical stirrer, here operated in 50 steps. This RC was shown to approximate isotropic diffusion starting from about $0.5 \mathrm{GHz}$ [6]. In order to apply (10) and (11), N+2 identical antennas are required. A total of six monocone antennas were used, as those pictured in Fig. 2, with two of them used as transmitter and receiver, while the rest were placed (not connected to any cable) on styrofoam supports, acting at stand-alone loading antennas. These were oriented in such a way to have their axis orthogonal to their closest neighbours, in order to minimize any direct coupling.

The reference average-power transmission, $\sigma_{o}^{2}(\nu)$, was measured without the four loading antennas, while keeping the styrofoam supports in place, in order to take into account

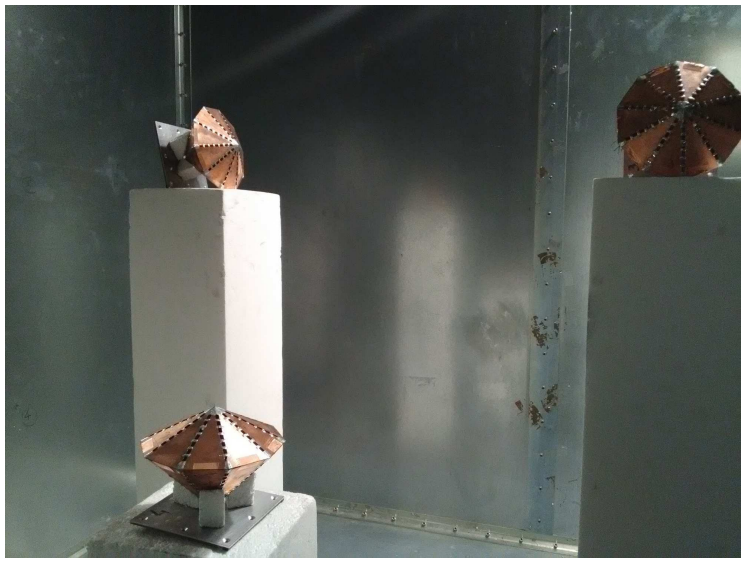

Fig. 2: Part of the experimental setup, with three of the loading antennas mounted on top of styrofoam supports.

eventual loading effects. Subsequent measurements for the loaded average-power transmission $\sigma_{N}^{2}(\nu)$ were carried out with the four loading antennas on the styrofoam supports, closing each antenna on identical impedances, characterized by a reflection coefficient $\Gamma_{c}(\nu)$ as defined with respect to a $50 \Omega$ reference impedance. For the sake of applying (5) and (6), the actual mismatch $\Gamma_{L}(\nu)$ observed from the antenna standpoint needs to be defined with respect to its own impedance, in general different from $50 \Omega$. According to [7, Sec. 4.4.4]

$$
\Gamma_{L}(\nu)=\frac{-\Gamma_{a}(\nu)+\Gamma_{c}(\nu)}{1-\Gamma_{c}(\nu) \Gamma_{a}(\nu)} .
$$

Power transmission $\sigma_{N}^{2}(\nu)$ was measured over the frequency range $0.6-6 \mathrm{GHz}$, with 60000 evenly spaced samples, using a Rohde \& Schwarz VNA, model ZVB8. Five different loading impedances were tested: open circuits (as SMA coaxial connectors), $50 \Omega$ loads, and coaxial attenuators left in open circuit, with attenuations equal to 1,3 and $6 \mathrm{~dB}$. The rationale behind these choices was to span a large panel of impedance mismatches. In particular the open-circuited attenuators simulate intermediate conditions between the case of an open-circuited antenna and a $50 \Omega$ impedance. Results in Fig. 3 show how the free-space mismatch $\Gamma_{a}(\nu)$ (found for $\Gamma_{c}=0$, i.e., for a $50 \Omega$ load) is translated into a different $\Gamma_{L}(\nu)$ as soon as relatively high values of $\left|\Gamma_{c}\right|$ are considered. Of particular interest is the appearance of oscillations in the lower frequency range, indicating that the denominator in (12) becomes partially resonant.

The resulting sets of $\sigma_{N}^{2}(\nu)$ were smoothed over a 100 $\mathrm{MHz}$ sliding window, since cavity-dominated losses result into asymptotically vanishing differences with respect to the unloaded case, $\sigma_{o}^{2}(\nu)$. The ratios $R(\nu)=\sigma_{o}^{2}(\nu) / \sigma_{N}^{2}(\nu)=$ $Q_{o}(\nu) / Q_{N}(\nu)$, for the 5 loading configurations, are shown in Fig. 4, indicating how changing $\Gamma_{L}(\nu)$ leads to a varying degree of antenna-related loading, not limited to the lower frequency range, as often assumed. The case of open-circuited loading antennas is interesting, as it was found to systematically result into $R(\nu)>1$, in contradiction with standard model (5), for which $R(\nu)=1$ whenever $\left|\Gamma_{L}(\nu)\right|=1$, inde- 


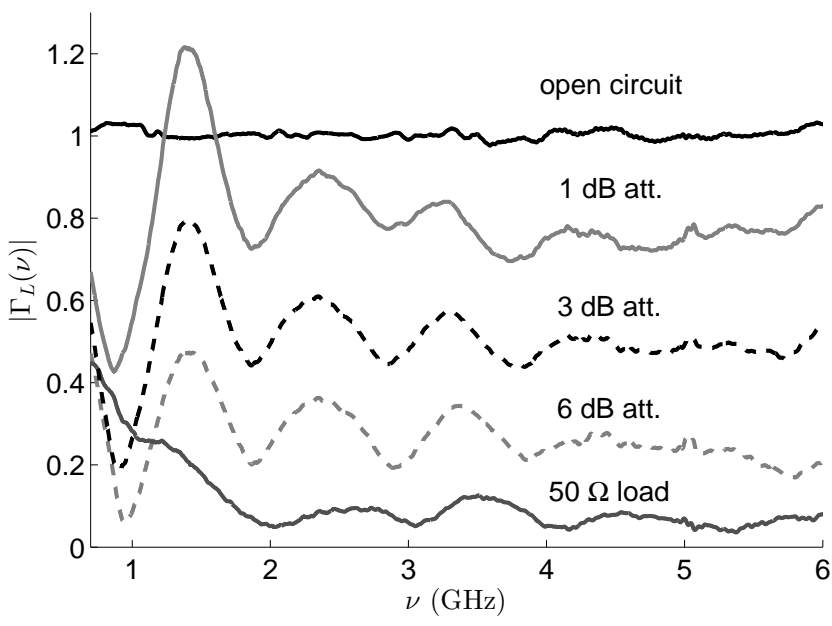

Fig. 3: Modulus of the reflection coefficients expected at the port of each loading antenna, as estimated from (12).

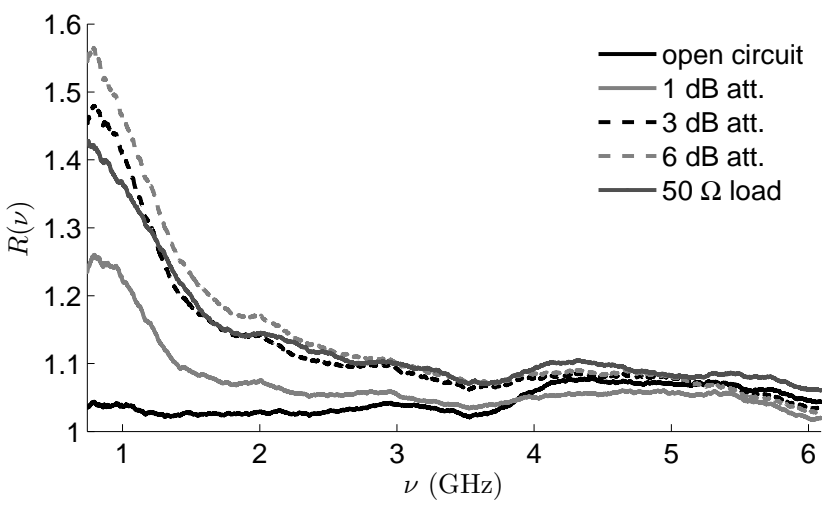

Fig. 4: Average power-transmission ratio $R(\nu)=$ $\sigma_{o}^{2}(\nu) / \sigma_{N}^{2}(\nu)$, measuring the increase in losses due to antenna-loading dissipation, for each antenna load.

pendently from $\eta_{a}(\nu)$, pointing to an underlying dissipation from the open-circuited antennas.

Estimates of $\eta_{a}(\nu)$ from (10) and (11) obtained from these data are shown in Fig. 5. It appears that standardmodel results are afflicted by a strong sensitivity to antenna loads, with oscillations in $\left|\Gamma_{L}(\nu)\right|$ translated into $\eta_{a}(\nu)$. The proposed model provides more consistent results, even though minor variations are observed between 1 and $2 \mathrm{GHz}$. Another measure of inconsistency with the standard model is that $\eta_{a}^{2}(\nu)$ in (11) had negative values, as well as $\eta_{a}(\nu)>1$, as visible in Fig. 5 below $2 \mathrm{GHz}$ for the strongly mismatched cases of an open-circuit and a $1 \mathrm{~dB}$ attenuator. These results confirm that the proposed model is more accurate than the standard one, as local differences are within a $\pm 10 \%$ range and physical constraints on $\eta_{a}(\nu)$ are respected. Finally, it can be noticed that the standard model inaccuracy translates into what appears to be an overall underestimation of $\eta_{a}(\nu)$, which could become an issue when testing antenna efficiencies in an RC.
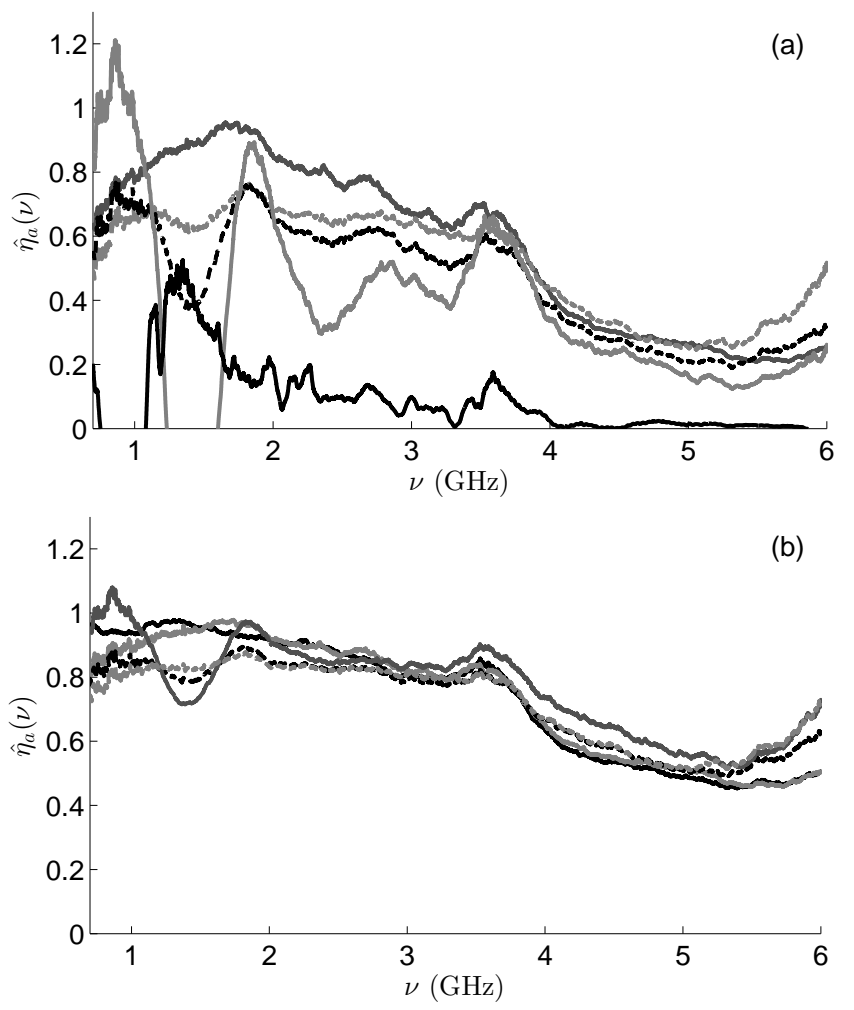

Fig. 5: Radiation efficiency as estimated from: (a) the standard antenna loading model, according to (11) and (b) the proposed model, according to (10). Refer to Figs. 3 and 4 for shade coding.

\section{CONCLUSION}

The proposed model for antenna/EUT dissipation within an RC was proven to be self-consistent in its predictions, as opposed to the standard one. These results are expected to be of interest in application of RCs where power loss is not dominated by cavity surfaces, with antennas/EUTs contributing to it, a situation known to occur in the lower frequency range of use of RCs.

\section{REFERENCES}

[1] D. Hill, "Electromagnetic Theory of Reverberation Chambers," National Institute of Standards and Technology, Tech. Rep. 1506, 1998.

[2] J. Ladbury and D. A. Hill, "Enhanced backscatter in a reverberation chamber: Inside every complex problem is a simple solution struggling to get out," in 2007 IEEE International Symposium on Electromagnetic Compatibility, July 2007, pp. 1-5.

[3] I. Junqua, P. Degauque, M. Liénard, and F. Issac, "On the power dissipated by an antenna in transmit mode or in receive mode in a reverberation chamber," IEEE Trans. Electromagn. Compat., vol. 54, no. 1, pp. 174180, Feb 2012

[4] C. L. Holloway, H. A. Shah, R. J. Pirkl, W. F. Young, D. A. Hill, and J. Ladbury, "Reverberation chamber techniques for determining the radiation and total efficiency of antennas," IEEE Trans. Antennas Propag., vol. 60 , no. 4, pp. 1758-1770, 2012.

[5] J. C. West, J. N. Dixon, N. Nourshamsi, D. K. Das, and C. F. Bunting, "Best practices in measuring the quality factor of a reverberation chamber," IEEE Trans. Electromagn. Compat., vol. PP, no. 99, pp. 1-8, 2017.

[6] A. Cozza, "Likelihood of meeting isotropic conditions in reverberation chambers from power transmission," IEEE Antennas Wireless Propag. Lett., vol. 16, pp. 2881-2884, 2017.

[7] J. Dobrowolski, Microwave network design using the scattering matrix. Artech House, 2010. 